\title{
Evaluación agronómica de familias de medios hermanos de lulo de Castilla, Solanum quitoense Lam.
}

\section{Agronomic evaluation of half sib families of lulo de Castilla, Solanum quitoense Lam.}

\author{
Liz Katherine Lagos Santander ; Franco Alirio Vallejo Cabrera ${ }^{2}$; Tulio César Lagos Burbano ${ }^{3}$; David Esteban Duarte Alvarado ${ }^{4}$
}

\begin{abstract}
${ }^{1}$ Ing. Agrónoma, M.Sc. Universidad de Nariño, Grupo de Investigación en Producción de Frutales Andinos. Pasto - Nariño, Colombia; e-mail: Iklagoss@unal. edu.co; (1) https://orcid.org/0000-0001-9932-0909

${ }^{2}$ Ing. Agrónomo, Ph.D. Universidad Nacional de Colombia, sede Palmira, Genética y Mejoramiento de Plantas. Palmira - Valle del Cauca, Colombia; e-mail: favallejoc@unal.edu.co; (D) https://orcid.org/0000-0002-2739-0745
\end{abstract}

${ }^{3}$ Ing. Agrónomo, Ph.D. Universidad de Nariño. Pasto - Nariño, Colombia; e-mail: tclago3@yahoo.com; (D) https://orcid.org/0000-0001-9222-4674

${ }^{4}$ Ing. Agrónomo. Universidad de Nariño, Grupo de Investigación en Producción de Frutales Andinos. Pasto - Nariño, Colombia; e-mail: david890223@ hotmail.com; (D) https://orcid.org/0000-0002-8896-3594

Cómo citar: Lagos Santander, L.K.; Vallejo Cabrera, F.A.; Lagos Burbano, T.C.; Duarte Alvarado, D.E. 2020. Evaluación agronómica de familias de medios hermanos de lulo de Castilla, Solanum quitoense Lam. Rev. U.D.C.A Act. \& Div. Cient. 23(1):e1334. http://doi. org/10.31910/rudca.v23.n1.2020.1334

Artículo de acceso abierto publicado por Revista U.D.C.A Actualidad \& Divulgación Científica, bajo una licencia Creative Commons CC BY-NC 4.0

Publicación oficial de la Universidad de Ciencias Aplicadas y Ambientales U.D.C.A, Institución de Educación Superior Acreditada de Alta Calidad por el Ministerio de Educación Nacional.

Recibido: Septiembre 3 de 2019

Aceptado Mayo 11 de 2020

Editado por: Ingeborg Zenner de Polanía

\section{RESUMEN}

El lulo (Solanum quitoense Lam.) es una especie frutal de gran importancia para el desarrollo de la fruticultura de la región natural Andina de Colombia; sin embargo, su cultivo presenta un bajo desarrollo tecnológico, debido posiblemente, a la escasa oferta de cultivares mejorados que, respondan a los limitantes abióticos y bióticos, que se presentan en los sistemas productivos de Colombia. El objetivo fue evaluar el comportamiento agronómico del rendimiento y calidad de fruto de 50 familias de medios hermanos de lulo, formadas a partir de un esquema de selección estratificada, en cuatro localidades del departamento de Nariño, Colombia. Se utilizó el diseño de Bloques Completos al Azar con tres repeticiones, cada repetición estuvo conformada por cuatro plantas, para un total de 600 unidades experimentales por municipio. Las variables evaluadas fueron rendimiento (RTO), pérdida de frutos por Neoleucinodes elegantalis, peso de fruto (PF), diámetro ecuatorial y polar, contenido de jugo (CJ), sólidos solubles totales (SST), acidez titulable (AC), índice de madurez (IM), pH, peso de pulpa más semilla (PPS) y peso de semilla (PS). Las familias de mejor comportamiento correspondieron a FMH28 y FMH22, para el municipio de Arboleda; FMH1, FMH7 y FMH25, para el municipio de San Pedro de Cartago y FMH28, FMH4, FMH36 y FMH29 para el municipio de Tangua, las cuales, presentaron rendimientos entre 6,86 a 15,81 tha ${ }^{-1}$, un PF entre 53,80 a 90,46g y SST entre 9,11 a $11,70^{\circ} \mathrm{Bx}$. Estos valores superaron a los promedios de la población 
evaluada en un $45,5 \%$, para RTO; para PF, en $11,41 \%$ y, para SST. en $10,50 \%$.

Palabras clave: fruticultura; naranjilla; comportamiento; selección; rendimiento; peso de fruto.

\section{ABSTRACT}

The lulo (Solanum quitoense Lam.) is a fruit of great importance for the development of fruit growing in the natural Andean region of Colombia; however, its cultivation presents a low technological development, possibly due to the scarce supply of improved cultivars that respond to the abiotic and biotic limitations that occur in the productive systems of Colombia. The objective was to evaluate the agronomic behavior of the yield and fruit quality of 50 half-brother families of lulo formed from a stratified selection scheme, in four localities of the department of Nariño, Colombia. The design of Complete Random Blocks with three repetitions was used; each repetition was made up of four plants for a total of 600 experimental units per municipality. The evaluated variables were yield (RTO), loss of fruits by Neoleucinodes elegantalis, fruit weight $(\mathrm{PF})$, equatorial and polar diameter, juice content (CJ), total soluble solids (SST), titratable acidity (AC), index of maturity (IM), $\mathrm{pH}$, pulp plus seed weight (PPS) and seed weight (PS). The best-behaving families corresponded to FMH28 and FMH22 for the municipality of Arboleda, FMH1, FMH7 and FMH25 for the municipality of San Pedro de Cartago and FMH28, FMH4, FMH36 and FMH29 for the municipality of Tangua, which had returns of 6,86 to 15.81 t.ha-1, a PF between 53.80 to $90.46 \mathrm{~g}$ and SST between 9.11 to $11.70^{\circ} \mathrm{Bx}$. These values exceeded the averages of the population evaluated by $45.5 \%$ for RTO, for PF in $11.41 \%$ and for SST in $10.50 \%$.

Keywords: Fruit growing; naranjilla; behavior; selection; yield; fruit weight.

\section{INTRODUCCIÓN}

El lulo Solanum quitoense Lam. es un frutal con un alto potencial productivo y ha sido catalogado desde muchos años atrás como un cultivo promisorio, a nivel nacional, debido a sus propiedades organolépticas (Muñoz et al. 2013). En diferentes zonas de la cordillera Andina Colombiana existen condiciones agroambientales óptimas para el desarrollo del cultivo; no obstante, este frutal aún se encuentra en proceso de domesticación y no se ha hecho uso de su variabilidad y biodiversidad. A causa de lo anterior, es necesario estudiar la variabilidad genética de la especie para adquirir el conocimiento de su potencial agronómico e industrial y, de esta manera, avanzar en la obtención de cultivares mejorados disponibles para que los productores enfrenten los diferentes problemas de campo, entre ellos, los fitosanitarios, que disminuyen el potencial productivo y generan un incremento en los costos de producción (Morillo Coronado et al. 2017; Silva et al. 2016).

En el departamento de Nariño, se presentan problemas fitosanitarios, como nemátodos (Meloidogyne spp.), hongos (Fusarium oxysporum, Phytophthora infestans) e insectos, como Neolencinodes elegantalis, que han causado pérdidas económicas, que van desde el 50 hasta el 90\% en cultivos comerciales (Ochoa et al. 2001; Lagos et al. 2015); también, Faustinus sp. y Alcidion sp., entre otros. Esto ha generado una baja sostenibilidad del cultivo, una disminución del potencial de rendimiento y deserción por parte de productores. Una opción ante la problemática planteada es implementar estrategias de mejoramiento genético, orientadas a la obtención de cultivares mejorados, con tolerancia a los factores bióticos mencionados, con alto rendimiento y que busquen aprovechar, de manera eficiente, la variabilidad genética de las colecciones de trabajo, tal como la selección familiar de medios hermanos en plantas alógamas (Peña et al. 2013).

En Colombia, los trabajos de investigación en mejoramiento genético en lulo son escasos. Se destaca, la obtención del cultivar de lulo La Selva, obtenido por CORPOICA (actualmente AGROSAVIA), en 1998 (Bernal et al. 1998). Según Medina et al. (2009), se ha reportado resistencia al nemátodo Meloidogyne incognita en materiales genéticos, derivados de hibridación inter específica, entre $S$. birtum y $S$. quitoense, al igual que en los clones que conforman el cultivar La Selva.

Por otro lado, la investigación en el cultivo de lulo ha girado en torno a estudios de filogenia y sus parientes silvestres (Bedoya-Reina \& Barrero, 2009); caracterización morfológica de 39 genotipos de la colección de lulo (S. quitoense), de la Universidad de Nariño (Riascos et al. 2012); caracterización molecular del gen de polifenolperoxidasa (Arias et al. 2013); comportamiento agronómico de injertos de lulo de castilla en patrones de Solanum spp. (Arizala et al. 2011; Ordoñez et al. 2012, Jurado et al. 2013); caracterización de la diversidad genética en el departamento de Boyacá (Morillo Coronado et al. 2017); crecimiento y desarrollo del fruto, en el municipio de San Antonio del Tequendama (Colombia) (Ochoa-Vargas et al. 2016) y reacción de genotipos de lulo a Meloidogyne spp. (Salazar-González \& Betancourth-Garcia, 2017).

A nivel internacional, algunas investigaciones, como la realizada por Silva et al. (2016), en el Ecuador, se basaron en evaluar la variabilidad genética obtenida, tomando parámetros importantes para el mejoramiento del cultivo, como grados Brix, color de pulpa y peso promedio de fruto, entre otros. Por otra parte, Gómez et al. (2008) evaluaron la adaptabilidad y la estabilidad del lulo, bajo condiciones de 23 fincas, en dos localidades de Nicaragua, donde demostraron que el cultivar evaluado se adapta a los ambientes con mayor índice ambiental y que se encuentran a una mayor altitud.

Entre los trabajos de mejoramiento genético en lulo de Castilla para Colombia, se destacan los ejecutados por Montes et al. (2010), quienes evaluaron la presencia de enfermedades causadas por hongos en 42 clones de lulo, en el municipio de Popayán y Lagos et al. (2015), quienes estudiaron el comportamiento de 50 familias de medios hermanos.

Dentro de los esquemas de selección en mejoramiento, se ha logrado que las poblaciones mejoradas lleguen a ser agronómicamente superiores y así, conservar la variabilidad genética en programas de mejoramiento genético sostenibles. En este sentido, la selección indirecta, tal como la Selección de Familia Medios Hermanos, se ha introducido para hacer más eficiente el incremento de la 
frecuencia de alelos favorables para caracteres de baja heredabilidad (Coutiño et al. 2008; Hallauer \& Carena, 2012; Peña et al. 2013) y la obtención de materiales mejorados. Estos esquemas tan usuales en cultivos tradicionales, como el maíz, la papa, el sorgo, entre otros, son incipientes en especies como el lulo, de ahí, que el objetivo de este trabajo fue evaluar 50 familias de medios hermanos (FMH) de lulo de Castilla, teniendo en cuenta su comportamiento agronómico del rendimiento y calidad de fruto, en cuatro localidades del departamento de Nariño, sur de Colombia.

\section{MATERIALES Y MÉTODOS}

Localización. La obtención de las 50 FMH, se llevó a cabo en dos localidades del departamento de Nariño: municipio de Yacuanquer, a N1 06 '55”, W77²4'04" y 2708m s.n.m. y municipio de La Florida, localizada a N1¹8'06" y W77²4'38” y 2.089 m s.n.m. La fase de evaluación y selección de las FMH, se realizó en los municipios de San Pedro de Cartago, ubicado a N1 $33^{\circ}$ '03', W7707'08” y $1.750 \mathrm{~m}$ s.n.m.; Tangua, a N1 ${ }^{\circ} 05^{\prime} 42^{\prime \prime}$, W77²3'39” y 2.000m s.n.m.; Arboleda, a N5³5’00”, W7509’00” y 2.100m s.n.m. y La Unión, a N1³6’18” y W7708'01" y $1.726 \mathrm{~m}$ s.n.m. Todos los municipios anteriores, se encuentran en la región alto Andina del departamento de Nariño.

Familias de medios hermanos (FMH). El grupo de investigación en Producción de Frutales Andinos de la Universidad de Nariño, introdujo en el 2008, semillas de plantas sobrevivientes de un cultivo de lulo de Castilla, S. quitoense, afectado por el hongo Fusarium sp., ubicado en el C.I. La Selva Agrosavia, Antioquia. En el semestre A, del 2013, dicha semilla se sembró en los municipios de la Florida y Yacuanquer, departamento de Nariño. Bajo un esquema de selección estratificada, se escogieron 50 plantas, por su estado sanitario, vigor y buena carga de fruto. De cada una de estas plantas, se tomó un fruto, se extrajo la semilla, que se identificó como una FMH, conformando así las $50 \mathrm{FMH}$.

Evaluación y selección de las 50 FMH de Lulo. En los municipios antes mencionados, se establecieron cuatro ensayos para evaluar el desempeño agronómico de las 50 FMH. Se utilizó el diseño de Bloques Completos al Azar con las $50 \mathrm{FMH}$, como tratamientos y tres repeticiones. Cada parcela experimental estuvo constituida por un surco de $8 \mathrm{~m}$ de largo. En él, se sembraron cuatro plantas con distancia entre ellas de 2,5m. La separación entre surcos (parcelas) fue de $3 \mathrm{~m}$. El área de la parcela experimental fue de $60 \mathrm{~m}^{2}$ y la superficie de la parcela útil de $22,5 \mathrm{~m}^{2}$, que correspondieron a tres plantas centrales, sobre las cuales, se realizaron las evaluaciones.

Variables evaluadas. Una vez las plantas llegaron a la fase productiva, a los 8 meses de establecimiento en campo, se registraron las variables: 1) Eje polar del fruto (DP) y eje ecuatorial del fruto (DE), que corresponden al promedio en mm de 12 frutos maduros de la segunda cosecha, provenientes de la parcela útil; 2) peso del fruto $(\mathrm{PF})$ en $\mathrm{g}$, de 12 frutos de la segunda cosecha provenientes de la parcela útil; 3) rendimiento (RTO), que se determinó en t.ha ${ }^{-1}$, con base en la producción de la parcela útil; 4) pérdida de frutos por N. elegantalis (PNEO), tomada mediante el conteo de frutos afectados con perforaciones causadas por las larvas y expresada en porcentaje del total de frutos cosechados. En postcosecha, teniendo en consideración el promedio de 12 frutos maduros provenientes de la parcela útil; 5) contenido de jugo (CJ), tomada en $\mathrm{ml}$ de cada fruto evaluado en laboratorio; 6) peso de pulpa más semilla (PPS), expresado en g, a partir de la extracción manual de la pulpa en laboratorio; 7) pH se determinó a través de un pH-metro LabNet; 8) acidez titulable (AC), a través del método de titulación potenciométrica; 9) sólidos solubles totales (SST), se obtuvieron con el método refractométrico. El valor de SST fue corregido por la acidez titulable, mediante la ecuación SSTc $=0,194 \times \mathrm{AC}+\mathrm{SST}$, donde SSTc $=$ SST corregidos, 10) índice de madurez (IM), que es igual a la relación entre los SSTcor y la acidez titulable.

Análisis estadístico. Para cada una de las variables evaluadas, se realizó el Análisis de Varianza combinado (ANDEVA), con el Software SAS ${ }^{2}$ 9.4, bajo un modelo mixto, donde las $50 \mathrm{FMH}$, se consideran de efecto fijo y las localidades, como efecto aleatorio. Cuando la interacción de las familias por ambiente ( $\mathrm{LxFMH})$ fuera significativa, el análisis se hizo con base en el comportamiento de las FMH en cada una de las localidades. Cuando la interacción LxFMH no fue significativa, se probó la hipótesis nula para los efectos simples (localidades, FMH).

En las variables donde la prueba de hipótesis permitió rechazar la hipótesis nula, se establecieron las diferencias entre las $\mathrm{FMH}$, teniendo en cuenta lo planteado por Antuna et al. (2003) y De la Cruz et al. (2010), quienes establecen que los valores superiores de una característica en particular son los que superan a la media general, más una vez el error estándar $(\mu+\sigma)$ o dos veces su correspondiente error estándar $(\mu+2 \sigma)$. En el caso de variables no deseables, como el peso de semilla por fruto y pérdida por $N$. elegantalis, los materiales genéticos más sobresalientes fueron aquellos que estuvieron por debajo de $\mu-\sigma$ o $\mu-2 \sigma$. Igualmente, todas las variables se sometieron al Análisis de Correlación simples de Pearson.

Con base en las medias obtenidas dentro y a través de localidades, se escogieron las mejores FMH, utilizando una presión de selección del 18\% y los mayores valores del índice de selección (IS), el cual, se estructuró con las variables RTO, PF, SSTc, IM, CJ y PNEO. Acorde con la metodología de Lagos et al. (2015), el primer paso para calcular el IS es estandarizar (E) los valores de cada una de las variables que componen el IS, mediante las siguientes ecuaciones:

$$
\begin{aligned}
E & =\frac{\left(X_{i}-\mu\right)}{\sigma} \\
\sigma & =\sqrt{\sigma^{2}} \\
\sigma^{2} & =\frac{\left(X_{i}-\mu\right)^{2}}{2}
\end{aligned}
$$

Dónde: $\mathrm{X}_{\mathrm{i}}=$ observación individual; $\mu=$ promedio general de cada variable; $\sigma^{2}=$ varianza; $\sigma=$ desviación estándar de la variable; $\mu=$ media poblacional y $\mathrm{N}=$ total de observaciones.

Por lo tanto, el IS aplicado fue el siguiente:

$$
\begin{gathered}
\mathrm{IS}=(\mathrm{RTO} \times 0,5)+(\mathrm{PF} \times 0,3)+(\mathrm{SSTc} \times 0,3)+(\mathrm{IM} \times 0,1) \\
+(\mathrm{CJ} \times 0,2)-(\mathrm{PNEO} \times 4)
\end{gathered}
$$




\section{RESULTADOS Y DISCUSIÓN}

El ANDEVA combinado (Tabla 1) mostró diferencias significativas entre localidades para todas las variables evaluadas. Según los análisis de suelo de cada localidad, se observan características contrastantes, como por ejemplo, en el contenido de material orgánica (MO), capacidad de intercambio catiónico (CIC) y Nitrógeno $(\mathrm{N})$, entre otros. Se destaca el municipio de Arboleda, con un contenido de MO de 11,1\%, considerado alto, teniendo en cuenta la temperatura promedio de la zona, que corresponde a $22^{\circ} \mathrm{C}$, presenta un $\mathrm{pH}$ adecuado de 6,29 y un grado textural del suelo Franco-Areno-Arcilloso, con una CIC de 34,0. Igualmente, el ANDEVA presenta diferencias significativas entre tratamientos, para las variables PF, SSTc y PPS $(p<0,05)$. La interacción Localidad $x$ FMH fue significativa para PF $(p<0,05)$ y altamente significativa para CJ, SST, AC, IM y RTO ( $<<0,01)$. En cuanto a los coeficientes de variación fenotípica (CVF) y genotípica (CVG), se categorizaron como bajos (0-10\%), medios (10-20\%) y altos (>20\%) (Abebe et al. 2017). Los CVF para las variables EP, DE, SST, AC, IM y pH, se consideran bajos y variaron entre $2,84 \%$, para el $\mathrm{pH}$ y $9,75 \%$, para DE. Para las variables PF, CJ y PPS, los CVF fueron moderados, desde $14,97 \%(\mathrm{PF})$ a $18,19 \%$ (CJ) y para las variables PNEO, PS y RTO, los CVF son altos, con valores entre 32,63\%, para PS y $37,02 \%$, para PNEO.

Según Abebe et al. (2017), la estrecha diferencia entre los CVF y CVG para las variables EP, DE, SST, IM y pH demostró que hay una baja influencia del ambiente para la expresión de dichas variables, de manera que la selección por el fenotipo será efectiva para mejorar el genotipo. Con relación a las variables PNEO, PF, CJ, AC, PPS, PS Y RTO, donde los CVF y CVG presentaron amplia diferencia, es posible que el ambiente afecte la expresión de las variables mencionadas.

Acorde con el Análisis de Correlación de Pearson (Tabla 2), se presentaron correlaciones $(\mathrm{r}$ ) positivas significativas entre PF y las variables PPS $\left(r=0,86^{*}\right), \mathrm{EP}\left(\mathrm{r}=0,52^{*}\right), \mathrm{DE}\left(\mathrm{r}=0,51^{*}\right)$ y $\mathrm{CJ}\left(\mathrm{r}=0,58^{*}\right)$. Esto demostró que existe una relación directa entre estas variables, por lo tanto, es posible mejorar el PF, si se direcciona la selección con base en cualquiera de los componentes del fruto de lulo citados (Gabriel et al. 2016).

Tabla 1. Cuadrados medios del ANDEVA para variables de rendimiento y calidad de fruto, evaluadas en 50 FMH de $S$. quitoense, a través de cuatro localidades de la región natural Andina del departamento de Nariño.

\begin{tabular}{|c|c|c|c|c|c|c|c|}
\hline FV & GL & PNEO & PF & EP & $\mathrm{DE}$ & CJ & SST \\
\hline LOC & 3 & $96938,5^{* *}$ & $11763,35^{* *}$ & $8,01 *$ & $15,71 * *$ & $14113,49 * *$ & $95,11^{* *}$ \\
\hline REP(Loc) & 8 & 254,79 & 305,50 & $1,2^{*}$ & $1,44^{*}$ & $869,99 *$ & $0,66^{* *}$ \\
\hline FMH & 49 & 125,54 & $452,8^{*}$ & 0,44 & 0,55 & 551,89 & $2,19 *$ \\
\hline LOC X FMH & 141 & 167,50 & $274,12^{*}$ & 0,50 & 0,64 & $520,9 * *$ & $1,34^{* *}$ \\
\hline ERROR & 321 & 139,66 & 208,15 & 0,49 & 0,61 & 370,91 & 0,24 \\
\hline$\mu \mathrm{g}$ & & 20,94 & 72,51 & 4,57 & 5,02 & 77,14 & 9,83 \\
\hline CVG \% & & 9,91 & 7,11 & 3,26 & 3,24 & 6,28 & 2,98 \\
\hline CVF \% & & 37,02 & 14,97 & 9,51 & 9,75 & 18,19 & 7,41 \\
\hline FV & GL & $\mathrm{AC}$ & IM & $\mathbf{P H}$ & PPS & PS & RTO \\
\hline LOC & 3 & $2,71 * *$ & $5,28 * *$ & $0,6^{* *}$ & $8182,22^{* *}$ & $369,7 * *$ & $433,84 * *$ \\
\hline REP(Loc) & 8 & 0,07 & 0,12 & $0,07 * *$ & 136,65 & 15,37 & $1,12^{*}$ \\
\hline FMH & 49 & 0,11 & 0,24 & 0,02 & $238,05^{*}$ & 17,18 & 10,91 \\
\hline LOC X FMH & 141 & $0,16^{* *}$ & $0,2^{* *}$ & 0,03 & 153,25 & 14,54 & $11,33^{* *}$ \\
\hline ERROR & 321 & 0,09 & 0,09 & 0,03 & 146,80 & 13,74 & 0,51 \\
\hline$\mu \mathrm{g}$ & & 3,41 & 2,90 & 3,56 & 46,46 & 7,40 & 5,60 \\
\hline CVG \% & & 0,90 & 3,18 & 0,99 & 8,40 & 13,41 & 1,90 \\
\hline CVF \% & & 6,83 & 9,44 & 2,84 & 17,53 & 32,63 & 34,67 \\
\hline
\end{tabular}

$\mathrm{CVF}=$ coeficiente de variación fenotípica $(\%) ; \mathrm{CVG}=$ Coeficiente de variación genético; $\mu \mathrm{g}=$ media general; $*=$ significativo a $\mathrm{p}=0,05 ;$ ** = significativo a $\mathrm{p}=0,01 ; \mathrm{ns}=$ no hay diferencias significativas o no significancia, perdida por Neoleucinodes elegantalis (PNEO), peso de fruto (PF), eje polar (EP), diámetro ecuatorial (DE), contenido de jugo (CJ), solidos solubles totales corregidos (SSTc), acidez titulable (AC), índice de madurez (IM), pH del jugo, peso de pulpa más semilla (PPS), peso de semilla (PS) y rendimiento (RTO). 
Tabla 2. Análisis de Correlación de Pearson para variables de rendimiento y calidad de fruta evaluadas en $50 \mathrm{FMH}$, a través de cuatro localidades de la región natural Andina del departamento de Nariño.

\begin{tabular}{|l|c|c|c|c|c|c|c|c|}
\hline Variable & PNEO & RTO & PF & EP & DE & IM & PPS & CJ \\
\hline PNEO & 1,00 & $-0,5^{*}$ & $\mathrm{~ns}$ & $\mathrm{~ns}$ & $\mathrm{~ns}$ & $\mathrm{~ns}$ & $\mathrm{~ns}$ & $\mathrm{~ns}$ \\
\hline PF & & & 1,00 & $0,52^{*}$ & $0,51^{*}$ & $\mathrm{~ns}$ & $0,86^{* *}$ & $0,58^{*}$ \\
\hline EP & & & & 1,00 & $0,97 *$ & $\mathrm{~ns}$ & $\mathrm{~ns}$ & $\mathrm{~ns}$ \\
\hline DE & & & & & 1,00 & $\mathrm{~ns}$ & $\mathrm{~ns}$ & $\mathrm{~ns}$ \\
\hline SSTc & & & & & & $0,63^{*}$ & $\mathrm{~ns}$ & $\mathrm{~ns}$ \\
\hline AC & & & & & & $-0,59 *$ & $\mathrm{~ns}$ & $\mathrm{~ns}$ \\
\hline IM & & & & & & 1,00 & $\mathrm{~ns}$ & $\mathrm{~ns}$ \\
\hline PPS & & & & & & & 1,00 & $0,58^{*}$ \\
\hline
\end{tabular}

$*$ = significancia con $\alpha<0,05 ; * *=$ significancia con $\alpha<0,01$, ns $=$ no significativo, pérdidas por Neoleucinodes elegantalis (PNEO), rendimiento (RTO), peso de fruto (PF), eje polar (EP) y diámetro ecuatorial (DE) del fruto, solidos solubles totales corregidos (SSTc), acidez tituable (AC), índice de madurez (IM), peso de pulpa más semilla (PPS) y contenido de jugo (CJ).

Los SSTc están correlacionados de manera significativa con el IM $\left(\mathrm{r}=0,63^{*}\right)$ y, a su vez, el IM presentó una correlación significativa negativa con la $\mathrm{AC}(\mathrm{r}=0,59 *)$, es decir, que a medida que aumenta el valor de AC disminuye el IM (Tabla 2). Gonzáles et al. (2013) determinaron que los SST están directamente relacionados con el grado de maduración del fruto y el contenido de ácidos orgánicos del mismo. Los SST, el IM y la AC son útiles al momento de determinar el punto óptimo de cosecha, dependiendo el mercado de distribución.

Como se puede observar en la tabla 2, entre el IM y la AC, se presentó una correlación significativa negativa $\left(r=-0.59^{*}\right)$. Según Almanza-Merchán et al. (2016), esta relación se da por la degradación de los ácidos orgánicos en la respiración, cuando ocurre el proceso de maduración que reduce la acidez, debido a la actividad de las hidrogenasas, ya que los ácidos orgánicos son utilizados como sustratos respiratorios para la síntesis de nuevos componentes, durante la maduración.

Teniendo en cuenta que se presentaron valores altos y significativos de correlación, solo se discutirán las variables SST, IM, CJ, PF y RTO, en las cuales, el ANDEVA combinado (Tabla 1) mostró un efecto de interacción LxFMH significativo. La interacción LxFMH significativa demostró una relación directa entre las diferencias ambientales y las diferencias de las FMH, para estas variables (García et al. 2002). En estos casos, es necesario analizar el comportamiento de las FMH evaluadas en cada una de las localidades, debido a la respuesta diferencial de los caracteres evaluados en los diferentes ambientes, tal como lo describen Sharifi et al. (2017), Nduwumuremyia et al. (2017), Abbott \& Pistorale (2011).

En la tabla 3, se encuentran las FMH con los mejores promedios del RTO, PF, SSTc, CJ e IM, evaluadas en las localidades de Arboleda, San Pedro de Cartago y Tangua. Conviene señalar, que el municipio de la Unión no fue un ambiente favorable para el potencial productivo y de poscosecha de las FMH evaluadas, de modo que los resultados de las variables en este municipio no se discutirán.

Rendimiento (RTO). En la tabla 3, se observó que para el carácter rendimiento, en la localidad de Arboleda, sobresalen las familias FMH34 (9,33t.ha $\left.{ }^{-1}\right)$, FMH20 (9,25t.ha $\left.{ }^{-1}\right)$, FMH35 (8,85t.ha $\left.{ }^{-1}\right)$, FMH28 (8,76t.ha $\left.{ }^{-1}\right)$, FMH44 (8,42t.ha $\left.{ }^{-1}\right)$, donde la media para esta localidad fue de 6,37 $\pm 1,61 \mathrm{t}^{-h^{-1}}$. San Pedro de Cartago presentó los mayores rendimientos con respecto a las demás localidades; los altos contenidos de fósforo, calcio, magnesio y potasio del suelo, favorecieron el desarrollo de las plantas. Se destacaron las familias FMH1 (15,81t.ha $\left.{ }^{-1}\right)$, FMH9 (13,59t.ha $\left.{ }^{-1}\right)$, FMH7 (13,52t.ha $\left.{ }^{-1}\right)$, FMH6 $\left(13,34 \mathrm{t}^{\mathrm{h}} \mathrm{h}^{-1}\right)$ y FMH25 $\left(13,02 \mathrm{t}^{\mathrm{h}} \mathrm{ha}^{-1}\right)$. Estas familias presentaron diferencias significativas con el resto de la población, dado que superaron la $\mu+\sigma$. Además, obtuvieron rendimientos superiores al promedio nacional, que corresponde a 9,45 $\mathrm{ha}^{-1}$ (AGRONET, 2019).

Lo anterior demostró el gran potencial que tienen estas familias evaluadas para esta localidad (Tosquy et al. 2005), al igual que en Tangua (Tabla 3), donde las familias FMH28 (10,78t.ha $\left.{ }^{-1}\right)$, FMH4 $\left(9,47 \mathrm{t}^{\mathrm{h}} \mathrm{h}^{-1}\right), \mathrm{FMH} 39$ (8,19t.ha $\left.{ }^{-1}\right)$, FMH19 $\left(8,08 \mathrm{t} \cdot \mathrm{ha}^{-1}\right)$ y FMH45 $\left(8,00\right.$ tha $\left.^{-1}\right)$, superaron la $\mu+2 \sigma$ y el promedio nacional; además, presenta condiciones agroclimáticas de interés, como textura de suelo Franco-arcillosa, pH adecuado de 6,04 y altos contenidos de materia orgánica, calcio y magnesio.

El RTO de lulo responde positivamente a la fertilización y el rendimiento potencial alcanza las 30 t.ha ${ }^{1}$. En los principales países productores de lulo, los rendimientos no sobrepasan las $8,5 \mathrm{t}^{-h \mathrm{ha}^{-1}}$, debido a las limitantes de carácter técnico que tienen que ver con el manejo agronómico, falencia en la selección de materiales mejorados, plagas, enfermedades, nutrición, falta de soporte en los procesos sistémicos de investigación y conocimiento del funcionamiento eco fisiológico de la especie (Gómez et al. 2013). 
Tabla 3. Promedios de las cinco mejores FMH para RTO en t/ha, PF, SSTc, CJ) e IM, obtenidas en las localidades de Arboleda, San Pedro de Cartago y Tangua (Nariño).

\begin{tabular}{|c|c|c|c|c|c|c|c|c|c|c|}
\hline Localidad & FMH & RTO & FMH & PF & FMH & SSTc & FMH & CJ & FMH & IM \\
\hline ARBOLEDA & 34 & $9,33^{*}$ & 49 & $88,74^{* *}$ & 15 & $9,92 * *$ & 49 & $99,57 * *$ & 15 & $3,54 * *$ \\
\hline ARBOLEDA & 20 & $9,25^{*}$ & 15 & $76,73^{*}$ & 23 & $9,76^{*}$ & 2 & $99,18^{* *}$ & 23 & $3,38^{* *}$ \\
\hline ARBOLEDA & 35 & $8,85^{*}$ & 6 & $75,33 *$ & 28 & $9,75^{*}$ & 3 & $89,57 *$ & 22 & $3,31 *$ \\
\hline ARBOLEDA & 28 & $8,76^{*}$ & 35 & $75,19 *$ & 18 & $9,57 *$ & 21 & $89,57 *$ & 49 & $3,21 *$ \\
\hline ARBOLEDA & 44 & $8,42^{*}$ & 29 & $72,98^{*}$ & 22 & $9,39 *$ & 16 & $86,69 *$ & 43 & $3,19 *$ \\
\hline MFS & & 8,92 & & 77,79 & & 9,68 & & 92,91 & & 3,33 \\
\hline Media (M) & & 6,37 & & 60,49 & & 8,97 & & 65,12 & & 2,86 \\
\hline $\mathrm{DE}$ & & 1,61 & & 9,95 & & 0,40 & & 12,94 & & 0,25 \\
\hline$\mu+\sigma$ & & 7,98 & & 70,44 & & 9,37 & & 78,06 & & 3,10 \\
\hline$\mu+2 \sigma$ & & 9,59 & & 80,40 & & 9,78 & & 91,00 & & 3,35 \\
\hline CARTAGO & 1 & $15,81 * *$ & 10 & $101,95^{* *}$ & 4 & $10,11^{*}$ & 31 & $99,58^{*}$ & 38 & $3,58^{*}$ \\
\hline CARTAGO & 9 & $13,59 * *$ & 27 & $100,46^{* *}$ & 14 & $10,0^{*}$ & 30 & $99,17 *$ & 14 & $2,95^{*}$ \\
\hline CARTAGO & 7 & $13,52 *$ & 17 & $94,92 *$ & 18 & $9,91 *$ & 14 & $97,92 *$ & 16 & $2,95^{*}$ \\
\hline CARTAGO & 6 & $13,34^{*}$ & 14 & $94,67 *$ & 3 & $9,75^{*}$ & 34 & $97,50^{*}$ & 48 & $2,93^{*}$ \\
\hline CARTAGO & 25 & $13,02^{*}$ & 31 & $93,79 *$ & 27 & $9,74^{*}$ & 17 & $95,00^{*}$ & 47 & $2,86 \mathrm{~ns}$ \\
\hline MFS & & 13,86 & & 97,16 & & 9,90 & & 97,83 & & 3,05 \\
\hline Media (M) & & 6,76 & & 76,30 & & 9,10 & & 74,73 & & 2,63 \\
\hline $\mathrm{DE}$ & & 3,41 & & 14,07 & & 0,61 & & 13,91 & & 0,25 \\
\hline$\mu+\sigma$ & & 10,17 & & 90,37 & & 9,72 & & 88,64 & & 2,88 \\
\hline$\mu+2 \sigma$ & & 13,58 & & 104,45 & & 10,33 & & 102,55 & & 3,12 \\
\hline TANGUA & 28 & $10,78^{* *}$ & 30 & $99,27^{*}$ & 15 & 12,31 ** & 13 & $178,75^{* *}$ & 2 & $4,12^{* *}$ \\
\hline TANGUA & 4 & $9,47 * *$ & 34 & $95,15^{*}$ & 2 & $12,23 * *$ & 11 & $105,83 \mathrm{~ns}$ & 36 & $3,98^{* *}$ \\
\hline TANGUA & 39 & $8,19^{*}$ & 7 & $95,04 *$ & 20 & $12,08^{*}$ & 7 & $103,33 \mathrm{~ns}$ & 3 & $3,49 *$ \\
\hline TANGUA & 19 & $8,08^{*}$ & 10 & $94,05^{*}$ & 14 & $12,07^{*}$ & 32 & $102,50 \mathrm{~ns}$ & 32 & $3,47^{*}$ \\
\hline TANGUA & 45 & $8,00^{*}$ & 11 & $93,60 *$ & 11 & $11,76^{*}$ & 25 & $102,04 \mathrm{~ns}$ & 4 & $3,43^{*}$ \\
\hline MFS & & 8,90 & & 95,42 & & 12,09 & & 118,49 & & 3,70 \\
\hline Media (M) & & 6,30 & & 83,50 & & 10,60 & & 91,26 & & 3,06 \\
\hline $\mathrm{DE}$ & & 1,52 & & 7,95 & & 0,76 & & 14,83 & & 0,32 \\
\hline$\mu+\sigma$ & & 7,82 & & 91,45 & & 11,36 & & 106,09 & & 3,37 \\
\hline$\mu+2 \sigma$ & & 9,34 & & 99,40 & & 12,12 & & 120,91 & & 3,69 \\
\hline
\end{tabular}

$*=$ Diferencias significativas al $\alpha=0,05 ; * *=$ Diferencias significativas al $\alpha=0,01 ;$ ns $=$ no hay diferencias significativas; $\sigma=$ desviación estándar; MFS = media de fracción seleccionada, rendimiento en $\mathrm{t} / \mathrm{ha}(\mathrm{RTO})$, peso de fruto en $\mathrm{g}(\mathrm{PF})$, solidos solubles totales corregidos en ${ }^{\circ}$ Brix (SSTc), contenido de jugo por fruto en $\mathrm{ml}$ (CJ) e índice de madurez (IM).

Variables postcosecha. Los frutos de las FMH fueron cosechados en un estado de madurez 4, según la NTC 5093 (ICONTEC, 2002), ya que Casierra-Posada et al. (2004) reportaron que este estado de maduración es óptimo para la evaluación de los variables que determinan la calidad, comparados con la evaluación de frutos en grado 5 , que equivale a la maduración de consumo.
En Arboleda (Tabla 3), los mayores valores de SSTc se registraron para la FMH15 $(9,92)$, que superó la $\mu+2 \sigma$ y FMH23 $(9,76)$, FMH28 (9,75), FMH18 $(9,57)$ y FMH22 $(9,39)$, que superaron la $\mu+\sigma(9,37)$. Para este municipio, se debe considerar el alto contenido de nitrógeno, fósforo y potasio, reportados en el análisis de suelos. Hernández-Fuentes et al. (2010) afirman que el contenido 
de SST se ve afectado por el contenido de nutrientes del suelo y una fertilización con fuentes de N-P-K adecuados, favorece la acumulación de ácidos orgánicos.

En la localidad de Cartago (Tabla 3), las FMH fueron FMH4 (10,11), FMH14 $(10,00)$, FMH18 $(9,91)$, FMH3 $(9,75)$ y FMH27 $(9,74)$, que superaron la $\mu+\sigma(9,72)$. En comparación con las localidades anteriormente descritas, Tangua, con 10,60 de SSTc, favoreció el incremento de esta variable, para las FMH, como se observó en la tabla 3.

En Tangua (Tabla 3), los valores altos de SSTc fueron 12,31 (FMH15), 12,23 (FMH2), 12,08 (FMH20), 12,07 (FMH14) y 11,76 (FMH11), valores por encima de los reportados: $8,24^{\circ}$ Brix (Silva

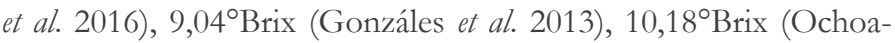
Vargas et al. 2016) y $7,6^{\circ}$ Brix (Almanza- Merchán et al. 2016). El incremento de los SST, se debe a que la hidrólisis del almidón y de los polisacáridos de la pared celular, generan azúcares solubles, tales como glucosa, fructosa y sacarosa, incidiendo de manera ascendente en el contenido de sólidos solubles y azúcares. La importancia de esta acumulación de azucares está relacionada con la calidad del fruto, en su forma consumible (Ochoa-Vargas et al. 2016).

Hay que mencionar que los SST aumentan a medida que transcurre el proceso de maduración (Almanza- Merchán et al. 2016). Este proceso no está relacionado con el $\mathrm{pH}$, según lo reportado por Casierra-Posada et al. (2004) y Galvis \& Herrera (1999), quienes observaron que, durante el periodo de maduración del lulo, el valor del pH es similar y oscila entre 2,9 y 3,2, entre el primero y el séptimo día de cosecha. Además, según los reportes de Casierra-Posada et al. (2004), se ha establecido que el valor del $\mathrm{pH}$ en jugo no debe ser tomado como una medida para establecer la madurez de los frutos.

Se encontró que los valores del IM (Tabla 3), en Arboleda, Cartago y Tangua, para FMH15, FMH23, FMH22, FMH49, FMH38, FMH14, FMH16, FMH48, FMH47, FMH36, FMH3, FMH32 у FHM4, oscilan entre 2,93 a 3,98. La FMH2 presentó el mayor IM, con un valor de 4,12, en la localidad de Tangua. Este IM está relacionado con el contenido de Vitamina C y el grado de madurez de los frutos, debido a que a mayor IM menor acidez, mayor contenido de azúcares e incremento de vitamina C (Gonzáles et al. 2013).

En cuanto al CJ, Arboleda (Tabla 3) presentó una media de $65,12 \pm 12,94 \mathrm{ml} /$ fruto; Cartago $74,73 \pm 13,91 \mathrm{~mL} /$ fruto y Tangua $91,26 \pm 14,83 \mathrm{~mL} /$ fruto. Las familias de mejor comportamiento para esta característica fueron FMH49 (99,57mL/fruto), FMH2 ( $99,18 \mathrm{~mL} /$ fruto), FMH3 (89,57mL/fruto), FMH21 (89,57mL/fruto) y FMH16 (86,69mL/fruto), en Arboleda. En Cartago sobresalen FMH31 (99,58mL/fruto), FMH30 (99,17mL/fruto), FMH14 $(97,92 \mathrm{~mL} /$ fruto), FMH34 $(97,50 \mathrm{~mL} /$ fruto) y FMH17 $(95,00 \mathrm{~mL} /$ fruto). En Tangua (Tabla 3), se presentaron los mejores promedios para esta variable, si se compara con las demás localidades. Se destacó en Tangua la FMH13, con 178,75mL/fruto.

Gonzáles et al. (2013) concluyeron que altos valores de CJ, SST, IM y Vitamina $C$ están relacionados con alto potencial de tipo industrial, calidad organoléptica y nutritiva del fruto de lulo; de ahí, la importancia de orientar los trabajos de investigación hacia el mejoramiento de las mismas.

En la tabla 4, se observan los promedios de las variables que componen el índice de selección (IS) de la fracción seleccionada $(18 \%)$, de las $50 \mathrm{FMH}$ de lulo de Castilla. Entre las familias que presentaron IS positivos, se destacaron FMH28, FMH22, FMH1, FMH7, FMH25, FMH28, FMH4, FMH36 у FMH29.

El RTO de la fracción seleccionada $\left(\bar{Y}_{s}\right)$ fue de $10,49 \mathrm{t} \mathrm{ha}^{-1}$, mientras que la media general fue de 5,32 t.ha $^{-1}$, por lo tanto, el diferencial de selección (DS) fue de 5,07t.ha ${ }^{-1}$, que indicó una ganancia del 45,15\%, en esta variable. El valor máximo entre los genotipos seleccionados correspondió a FMH1 con 15,81 tha ${ }^{-1}$. Estos resultados mostraron la posibilidad de avanzar en las ganancias genéticas del RTO de esta población, lo que concuerda con Vallejo \& Estrada (2016), quienes afirmaron que el mejoramiento poblacional consiste en estudiar el material básico, su composición y su estructura genética, con miras a incrementar el progreso en la selección a corto o largo plazo.

Lo anterior permite obtener nuevas poblaciones con mayor RTO después de cada ciclo de selección y que dicho incremento, se debe a que los individuos seleccionados poseen genes superiores que, al recombinarse al azar, producen genotipos de mayor producción, por lo tanto, se espera que la población sea más productiva en promedio que la anterior (Lagos et al. 2015).

El PF de las FMH seleccionadas osciló entre 53,80 y 90,46g. El mayor PF lo obtuvo la familia FMH36, con 90,46g y el DS fue de $7,98 \mathrm{~g}$, que representó una ganancia del 10,25\%. Los SSTc oscilaron entre 9,25 y 12,51, donde la familia FMH49 mostró el mayor contenido. El DS en los SSTc fue 1,0, que representa una ganancia del $9,51 \%$.

Por último, teniendo en cuenta el promedio de la fracción seleccionada de $11,87 \%$, la PNEO mostró un DS del 8,90\% respecto a la población de las $50 \mathrm{FMH}$. A pesar de observarse diferencias significativas, las ganancias en la disminución del PNEO fueron del 42,85\%, lo cual, puede representar una disminución en los costos de control de la plaga. Estas ganancias, se pueden mejorar con prácticas culturales y preventivas, como recolección de frutos afectados o aplicaciones de controladores biológicos (Carmona et al. 2006 y Gómez et al. 2013). Las familias FMH28, FMH22, FMH1, FMH7, FMH25, FMH28, FMH4, FMH36, FMH29 у FMH49 presentaron los mayores valores en el índice de selección. Estas familias mostraron los mejores resultados en cuanto a rendimiento, peso de fruto, sólidos solubles totales corregidos y menores daños causados por el pasador de fruto.

Se concluye que la evaluación y selección de familias de medios hermanos, como método de mejoramiento genético aplicado al cultivo de lulo, permite identificar cultivares sobresalientes en cuanto a rendimiento y calidad de fruto, que podrían ser usados para continuar con el proceso de mejoramiento genético en la especie. 
Tabla 4. Promedio de las variables que componen el índice de selección (IS) de la fracción seleccionada (18\%) de las 50 FMH de lulo de Castilla, evaluadas en cuatro ambientes de la región andina del departamento de Nariño.

\begin{tabular}{|l|c|c|c|c|c|c|c|c|}
\hline \multicolumn{1}{|c|}{ Localidad } & FMH & RTO & PF & SST & IM & CJ & PNEO & IS \\
\hline ARBOLEDA & $\mathbf{2 8}$ & 8,76 & 65,75 & 9,75 & 2,87 & 71,25 & 14,02 & $\mathbf{2 . 0 2}$ \\
\hline ARBOLEDA & $\mathbf{2 2}$ & 8,00 & 53,80 & 9,39 & 3,31 & 67,08 & 15,15 & $\mathbf{1 , 2 1}$ \\
\hline CARTAGO & $\mathbf{1}$ & 15,81 & 77,26 & 9,25 & 2,77 & 80,00 & 11,06 & $\mathbf{1 , 6 0}$ \\
\hline CARTAGO & $\mathbf{7}$ & 13,52 & 81,56 & 9,32 & 2,74 & 74,74 & 8,14 & $\mathbf{1 , 5 3}$ \\
\hline CARTAGO & $\mathbf{2 5}$ & 13,02 & 82,11 & 9,11 & 2,49 & 78,75 & 6,93 & $\mathbf{1 , 4 3}$ \\
\hline TANGUA & $\mathbf{2 8}$ & 10,78 & 81,43 & 11,37 & 2,93 & 84,17 & 2,77 & $\mathbf{1 , 9 2}$ \\
\hline TANGUA & $\mathbf{4}$ & 9,47 & 82,92 & 11,31 & 3,43 & 96,77 & 4,24 & $\mathbf{1 , 7 4}$ \\
\hline TANGUA & $\mathbf{3 6}$ & 7,28 & 90,46 & 11,49 & 3,98 & 77,83 & 0,00 & $\mathbf{1 , 6 1}$ \\
\hline TANGUA & $\mathbf{2 9}$ & 6,86 & 82,48 & 11,70 & 3,13 & 96,67 & 5,09 & $\mathbf{0 , 8 5}$ \\
\hline $\bar{Y}_{s}$ & & 10,39 & 77,53 & 10,30 & 3,07 & 80,81 & 7,49 & \\
\hline$\mu g$ & & 5,32 & 69,90 & 9,52 & 2,82 & 74,39 & 20,77 & \\
\hline DS & 5,07 & 7,63 & 0,78 & 0,26 & 6,42 & $-13,28$ & \\
\hline
\end{tabular}

$\bar{Y}_{s}=$ media de la fracción seleccionada; $\mu=$ media general de las $50 \mathrm{FMH} ; \mathrm{DS}=$ diferencial de selección $\left(\bar{Y}_{s}-\mu \mathrm{g}\right)$.

Conflictos de intereses: El manuscrito fue preparado y revisado con la participación de todos los autores, quienes declaramos que no existe ningún conflicto de intereses que ponga en riesgo la validez de los resultados presentados. Financiación: Este estudio fue financiado por el Fondo de ciencia, tecnología e innovación del Sistema General de Regalías.

\section{REFERENCIAS}

1. ABBOTT, L.; PISTORALE, S. 2011. Análisis de la estabilidad y adaptabilidad de caracteres de interés agronómico en genotipos selectos de cebadilla criolla (Bromus catharticus). Agriscientia. 26(2):109-117. https://doi. org/10.31047/1668.298x.v28.n2.2788

2. ABEBE, T.; ALAMEREW, S.; TULU, L. 2017 Genetic variability, heritability and genetic advance for yield and its related traits in rainfed lowland rice (Oryza sativa L.) Genotypes at Fogera and Pawe, Ethiopia. Adv. Crop Sci. Tech. 5:272. https://doi.or/10.4172/2329-8863.1000272

3. AGRONET. 2019. Red de Información y Comunicación del Sector Agropecuario de Colombia: Reporte: Área, producción y rendimiento nacional por cultivo. Disponible en: https://www.agronet.gov.co/estadistica/Paginas/ home.aspx?cod=1

4. ALMANZA-MERCHÁN, P.; VELANDIA, J.; TOVAR, Y. 2016. Propiedades fisicoquímicas durante el crecimiento y desarrollo de frutos de lulo (Solanum quitoense Lam.) Rev. Col. Ciencias Hortícolas. 10(2):222-231. https://doi. org/10.17584/rcch.2016v10i2.5065
5. ANTUNA, G.; RINCÓN, S.; GUTIÉRREZ, R.; RUIZ, T.; BUSTAMANTE, G. 2003. Componentes genéticos de caracteres agronómicos y de calidad fisiológica de semillas en líneas de maíz. Rev. Fitotecnia Mexicana. 26:11-17.

6. ARIAS, D.; MEJÍA, C.; RENGIFO, L.; NAVIA, C. 2013. Molecular characterization of the polyphenol oxidase gene in lulo (Solanum quitoense Lam.) var. Castilla. Braz. J. Plant Physiol. 24(4):261-272. http://dx.doi.org/10.1590/S167704202012000400005

7. ARIZALA, M.; MONSALVO, Á.; GARCÍA, C.B.; GONZÁLEZ, C.S.; LAGOS, T.C. 2011. Evaluación de solanáceas silvestres como patrones de lulo (Solanum quitoense Lam) y su reacción a Fusarium sp. Rev. Ciencias Agrícolas. 28(1):147-160.

8. BEDOYA-REINA, O.; BARRERO, L.S. 2009. Filogenia de lulo, tomate de árbol y sus parientes silvestres. Ciencia \& Tecnología Agropecuaria. 10(2):180-190. https://doi. org/10.21930/rcta.vol10_num2_art:140

9. BERNAL, J.; LONDOÑO, M.; FRANCO, G.; LOBO, M. 1998.Lulo la selva En: ICA-CORPOICA. Primer material de lulo mejorado para Colombia. Rio Negro, Antioquia: CORPOICA. $8 \mathrm{p}$.

10. CARMONA, R.; COOMAN, A.; GÓMEZ, D.; FUENTES, L.; NIÑO, N.; ESPINOZA, L.; CUELLAR, J.; MEDINA, A.; ESCOBAR, H. 2006. Lulo: El Cultivo. Bogotá, Fundación Universidad Jorge Tadeo Lozano, ISBN 9589029-79-5. 100p. 
11. CASIERRA-POSADA, F.; GARCIA, J.; LÜDDERS, P. 2004. Determinación del punto óptimo de cosecha en el lulo (Solanum quitoense Lam.) Var. quitoense y septentrionale. Agron. Col. 22(1):32-39.

12. COUTIÑO, B.; SÁNCHEZ, G.; VIDAL, V. 2008. Selección entre y dentro de familias de hermanos completos de maíz en Chiapas, México. Rev. Fitotecnia Mexicana. 31(2):115123.

13. DE LA CRUZ, E.; CASTAÑÓN-NÁJERA, N.; BRITOMANZANO, A.; GÓMEZ-VÁZQUEZ, V.; ROBLEDO, T.; LOZANO, R. 2010. Heterosis y aptitud combinatoria de poblaciones de maíz tropical. Internal J. Experimental Botany. 79:11-17.

14. GABRIEL, J.; ANGULO, A.; VELASCO, J.; GUZMÁN, R. 2016. Adaptación de híbridos de tomate indeterminado Solanum lycopersicum L. (Mill.) bajo condiciones de invernadero. J. Selva Andina Res. Soc. 7(2):47-65. https:/ / doi.org/10.36610/j.jsars.2016.070200047

15. GALVIS, J.; HERRERA, A. 1999. El lulo Solanum quitoense Lam. Manejo de Poscosecha. Convenio SENA-Universidad Nacional de Colombia, Bogotá. 59p.

16. GARCÍA, J.; LÓPEZ, J.; MOLINA, J.; CERVANTES, T. 2002. Selección masal visual estratificada y de familias de medios hermanos en una cruzada intervarietal F2 de maíz. Rev. Fitotecnia Mexicana. 25(4):387-391.

17. GÓMEZ, F.; TREJO L.; GARCÍA J.; MORALES V. 2013. Lulo (Solanum quitoense Lamarck) como nuevo elemento del paisaje en México: germinación y crecimiento en sustratos orgánicos. Rev. Mexicana de Ciencias Agrícolas. Pub. Esp. 5:877-887.

18. GÓMEZ, O.; OROZCO, P.; CENTENO, B. 2008. Adaptabilidad y estabilidad de naranjilla (Solanum quitoense Lam. var. septentrionale) y cocona (Solanum sessiliflorum Dunal var. sessiliflorum) en fincas de pequeños agricultores de granos básicos en Jinotega. La Calera. 8(11):9.

19. GONZÁLES, D.; ORDOÑEZ, L.; VANEGAS, M.; VÁSQUEZ, H. 2013. Cambios en las propiedades fisicoquímicas de frutos de lulo (Solanum quitoense Lam.) cosechados en tres grados de madurez. Acta Agronómica. 63(1):11-17. https://doi.org/10.15446/acag.v63n1.31717

20. HALLAUER, A.; CARENA, M. 2012. Recurrent selection methods to improve germplasm in maize. Maydica. 57:266283.

21. HERNÁNDEZ-FUENTES, A.; CAMPOS, R.; PINEDOESPINOZA, J. 2010. Comportamiento poscosecha de pimiento morron (Capsicum annum L.) var. california por efecto de la fertilización química y aplicación de lombrihumus. Rev. Iberoam. Tecnología Postcosecha. 11(1):82-91.

22. INSTITUTO COLOMBIANO DE NORMAS TECNICAS, ICONTEC. 2002. Frutas frescas. Lulo de Castilla. Especificaciones. Bogotá, ICONTEC. 19p.

23. JURADO, J.C.; JERÉZ, L.J.; LAGOS, T.C.; BENAVIDES, C.A. 2013. Comportamiento agronómico de injertos de lulo de Castilla Solanum quitoense Lam. en patrones de Solanum spp. Rev. Ciencias Agrícolas. 30(1):54-64.

24. LAGOS, T.C.; APRAEZ, J.; LAGOS, L.K.; DUARTE, D. 2015. Comportamiento de 50 familias de medios hermanos de Solanum quitoense Lam. bajo selección recurrente. Revista Temas Agrarios. 20(2):19-29. http://doi.org/10.21897/ rta.v20i2.755

25. MEDINA, C.; LOBO, A.; MARTINEZ, B. 2009. Revisión del estado del conocimiento sobre la función productiva de lulo (Solanum quitoense Lam.) en Colombia. Corpoica Cienc. Tecnol. Agropecu. 10(2):167-179. https://doi. org/10.21930/rcta.vol10_num2_art:139

26. MONTES, C.; MUÑOZ, A.; TERÁN, V.; PRADO, F.; MAGALLY, A. 2010. Evaluación de patógenos en clones de lulo (Solanum quitoense Lam.) Acta Agron. 59(2):144-154.

27. MORILLO CORONADO, A.C.; RODRÍGUEZ FAGUA, A. DEL P.; MORILLO CORONADO, Y. 2017. Characterization of lulo (Solanum quitoense Lam.) genetic diversity in the department of Boyaca, Colombia. Acta Agron. 66(3):430-435. https://doi.org/10.15446/acag. v66n3.58997

28. MUÑOZ, J.; RODRÍGUEZ, L.; BERMÚDEZ, L. 2013. Análisis de competitividad del sistema de producción de lulo (Solanum quitoense Lam.) en tres municipios de Nariño. Rev, Col. Ciencias Hortícolas. 7(2):173-185.

29. NDUWUMUREMYIA, A.; MELISA, R.; SHANAHANA, P.; THEODOREB, A. 2017. Interaction of genotype and environment effects on important traits of cassava (Manihot esculenta Crantz). The Crop J. 5:373-386.

30. OCHOA, J.; GALARZA, V.; ELLIS, M. 2001. Diagnostic of naranjilla diseases in the Pastaza valley of Ecuador. In: Integrated Pest Management Collaborative Research Program (IPM-CRSP). Sixth Annual Report. Virginia Tech. Ed. Springer. (USA). p.267-270.

31. OCHOA-VARGAS, L.; BALAGUERA-LOPEZ, E.; ARDILAROA, G.; PINZON-SANDOVAL, E.; ALVAREZHERRERA, J. 2016. Crecimiento y desarrollo del fruto de lulo (Solanum quitoense Lam.) en el municipio de San Antonio 
del Tequendama (Colombia). Corpoica Cienc Tecnol Agropecuaria. 17(3):347-359. https://doi.org/10.21930/ rcta.vol17_num3_art:512

32. ORDOÑEZ, C.; GÓMEZ, H.; JURADO, H.R.; LAGOS, T.C. 2012. Evaluación de un sistema de propagación vegetativa mediante esquejes en lulo silvestre Solanum birtum Vahl, S. marginatum Lf, S. sessiliflorum Dun, S. mammosum L. y $S$. umbellatum Mill. Revista de Ciencias Agrícolas. 29(1):29-41.

33. PEÑA, A.; GUERRERO, H.; RODRIGUEZ, J.; SAHAGUN, J.; MAGAÑA, N. 2013. Selección temprana en familias de medios hermanos maternos de tomate de cascara de la raza Puebla. Revista Chapingo, Serie Horticultura. 19(1):5-13. http://dx.doi.org/10.5154/r.rchsh.2012.01.18

34. RIASCOS, M.; SANTACRUZ, A.; LAGOS, T.C.; CHECA, O. 2012. Caracterización morfológica de 39 genotipos de la colección de lulo (Solanum quitoense Lam.) de la Universidad de Nariño. Revista de Ciencias Agrícolas. 29(1):57-69.

35. SALAZAR-GONZÁLEZ, C.; BETANCOURTH-GARCIA, C. 2017. Reacción de genotipos de lulo (Solanum quitoense Lam.) a Meloidogyne spp. en condiciones de campo. Corpoica Cienc Tecnol Agropecuaria, 18(2):295-306. http://dx.doi. org/10.21930/rcta.vol18_num2_art:629
36. SAS Institute 2013. Base SAS® 9.4. Software. SAS Institute Inc. Cary, NC, USA.

37. SHARIFI, P.; AMINPANAH, H.; ERFANI, R.; MOHADDESI A.; ABBASIAN, A. 2017. Evaluation of Genotype $\times$ Environment Interaction in Rice Based on AMMI Model in Iran. Rice Science. 24(3):173-180. https://doi. org/10.1016/j.rsci.2017.02.001

38. SILVA, W.; GÓMEZ, P.; VIERA, W.; SOTOMAYOR, A.; VITERI P.; LENIN, R. 2016. Selección de líneas promisorias de naranjilla para mejorar la calidad de fruta. Ecuador es Calidad: Revista Ecuatoriana. 3:23-30. https://doi.org/10.36331/revista.v3i1.21

39. TOSQUY, O.; PALAFOX, A.; SIERRA, M.; ZAMBDA, A.; MARTINEZ, R.; GRANADOS, R. 2005. Comportamiento agronómico de híbridos de maíz en dos municipios de Veracruz, México. Agronomía Mesoamericana. 16(1):7-12. https://doi.org/10.15517/am.v16i1.5177

40. VALLEJO, F.; ESTRADA, E. 2016. Mejoramiento Genético de Plantas. Ed. Universidad Nacional de Colombia. (Palmira). 268p. 